\title{
Species prevalence and antibacterial resistance of enterococci isolated in Kuwait hospitals
}

\author{
Edet E. Udo, ${ }^{1}$ Noura Al-Sweih, ${ }^{1}$ Oludotun A. Phillips ${ }^{2}$ and Tulsi D. Chugh ${ }^{1}$

\begin{abstract}
Department of Microbiology, Faculty of Medicine ${ }^{1}$ and Department of Pharmaceutical Chemistry, Faculty of Pharmacy², Kuwait University, PO Box 24923, Safat 13110, Kuwait
\end{abstract}

Correspondence

Edet E. Udo

edet@hsc.kuniv.edu.kw

Received 19 April 2002

Accepted 1 October 2002

\begin{abstract}
This study investigated the species prevalence and antibacterial resistance among enterococci isolated in Kuwait hospitals. They consisted of 415 isolates of Enterococcus faecalis (85.3\%), Enterococcus faecium (7.7\%), Enterococcus casseliflavus (4.0\%), Enterococcus avium (1.2\%), Enterococcus durans (1.0\%), Enterococcus gallinarium (0.5\%) and Enterococcus bovis (0.2\%) isolated from urine (36.6\%), blood (10.4\%), wound swabs $(11.0 \%)$, stool samples (12.0\%), high vaginal swabs (9.0\%), endocervical swabs (3.0\%) and miscellaneous sources (18.0\%). All of them were susceptible to linezolid. Fifty-two (12.5\%) isolates were ampicillin resistant but none of them produced $\beta$-lactamase. They were resistant to erythromycin (63.3\%), tetracycline (60.5\%), ciprofloxacin (40.0\%), chloramphenicol (28.0\%), vancomycin (2.6\%), and teicoplanin (2.6\%). Fourteen, 19 and $20 \%$ of them expressed high-level resistance to gentamicin, kanamycin and streptomycin, respectively. All of the vancomycin-resistant strains carried the vanA phenotype and genotype. There was no evidence of clonal spread of the vancomycin-resistant isolates.
\end{abstract}

\section{INTRODUCTION}

Enterococci are members of the normal flora in the gut of humans and animals but have become increasingly important as hospital-acquired pathogens. They have been associated with infections of the urinary tract, post-surgical wounds, septicaemia, endocarditis and meningitis (Cetinkaya et al., 2000; Jones et al., 1997; Woodford et al., 1993; Huycke et al., 1998). They have a remarkable ability to adapt to exposure to antibacterials maintaining intrinsic resistance to penicillins and low-level resistance to aminoglycosides and a tremendous capacity to acquire resistance to other antibacterials including high-level resistance to aminoglycosides and glycopeptides (Quale et al., 1996; Donskey et al., 1999; Nelson et al., 2000). Vancomycin-resistant enterococci (VRE) have caused outbreaks in many centres in the USA (Cetinkaya et al., 2000; Donskey et al., 1999; Quale et al., 1996) and are recognized as an important resistant pathogen in Europe (Reinert et al., 1999; Schouten et al., 2000; Woodford et al., 1993) and (Toutouza et al., 2001). Infections caused by VRE are associated with severe adverse outcomes such as extended length of hospital stay, increased cost and increased mortality due to the reduction in the choices of antibacterials available to treat such infections (Linden et al., 1996; Huycke et al., 1998). Despite the increasing reports of VRE in different countries, the prevalence of VRE in Kuwait hospitals was unknown. A recent study of antibacterial resistance of enterococci isolated in a teaching hospital revealed a low prevalence of glycopeptide

Abbreviation: VRE, vancomycin-resistant enterococci. resistance (E. E. Udo, unpublished data). However, the situation in other hospitals remained uncertain. This paper reports on a study conducted to investigate the prevalence of antibacterial resistance in enterococci isolated from clinical samples in five hospitals in Kuwait.

\section{METHODS}

Enterococcus isolates. A total of 415 isolates was obtained from different clinical samples between January 1999 and 31 December 2001 in Mubarak Al-Kabeer (206), Amiri (63), Adan (28), Ibn Sina (46) and Maternity (74) hospitals in Kuwait. They were cultured from urine, wound swabs, blood, endotracheal secretions, cervical swabs, high vaginal swabs and ear swabs. Stool samples were inoculated into enterococcal broth, incubated overnight at $35^{\circ} \mathrm{C}$ and subcultured onto brain-heart infusion agar (BHIA) containing $6 \mu \mathrm{g}$ vancomycin $\mathrm{ml}^{-1}$ (Jordens et al., 1994; Ieven et al., 1999) and onto BHIA without vancomycin to recover vancomycin-susceptible isolates. The isolates were identified to the genus and species level by cultural characteristics, Gram's stain, catalase test, bile aesculin reactions and by biochemical tests using API Strep (bioMérieux).

Susceptibility testing. The disk diffusion method on MuellerHinton agar (National Committee for Clinical Laboratory Standards, 2000) was used to detect resistance to tetracycline, erythromycin, chloramphenicol, ampicillin, ciprofloxacin, gentamicin, kanamycin, streptomycin, vancomycin and teicoplanin. Detection of high-level aminoglycoside resistance was performed with disks containing gentamicin $(120 \mu \mathrm{g})$, kanamycin $(200 \mu \mathrm{g})$ and streptomycin $(300 \mu \mathrm{g})$. Results were read after incubation at $35^{\circ} \mathrm{C}$ for $24 \mathrm{~h}$ and after $48 \mathrm{~h}$ for streptomycin. MICs for vancomycin and teicoplanin were determined using E-test strips (AB Biodisk). MICs for gentamicin, kanamycin, streptomycin, tobramycin, amikacin and linezolid were determined by 
the agar dilution method. Enterococcus faecalis strain ATCC 29212 was used as a sensitive control. Linezolid powder was a gift from Pharmacia.

Detection of $\beta$-lactamase production. $\beta$-Lactamase production was detected with nitrocefin (Oxoid) according to the manufacturer's instructions. Nitrocefin solution $(5 \mu \mathrm{l})$ was dropped onto a loopful of pure overnight growth placed on a filter membrane. The development of a red colour within 60 s indicated a positive result. Staphylococcus aureus strain ATCC 29213 was used as a positive control.

Detection of vancomycin-resistance determinants. Genes encoding the vancomycin-resistance determinants vanA and vanB were investigated by PCR using specific primers as published in the literature (Dutka-Malen et al., 1995; Miele et al., 1995). The primers were VanA (VA1, 5' -GGG AAA ACG ACA ATT GC-3' and VA2, 5' -GTA CAA TGC GGC CGT TA-3'), VanB (VA3, 5' -CCC GAA TTT CAA ATG ATT GAA AA-3', and VA4, 5' -CGC CAT CCT CCT GCA AAA-3' $)$. Amplification was performed using a kit from Gibco-BRL. DNA for PCR was isolated as described previously (Udo \& Dashti, 2000) except that lysozyme $\left(1 \mathrm{mg} \mathrm{ml}^{-1}\right)$ was used instead of lysostaphin in the lysis solution. The amplification mixture consisted of $45 \mu$ Supermix $(22 \mathrm{mM}$ Tris/ $\mathrm{HCl}$, pH 8.4, $55 \mathrm{mM} \mathrm{KCl}, 1.65 \mathrm{mM} \mathrm{MgCl}_{2}, 220 \mu \mathrm{M}$ each dNTP, $22 \mathrm{U}$ recombinant Taq DNA polymerase $\mathrm{ml}^{-1}, 3 \mu \mathrm{l}$ bacterial DNA and $2 \mu \mathrm{l}$ primer solution in a total reaction volume of $50 \mu \mathrm{l}$. A Perkin Elmer 9600 thermocycler was programmed for 32 cycles with the following parameters: denaturation at $94^{\circ} \mathrm{C}$ for $3 \mathrm{~min}$, annealing at $60^{\circ} \mathrm{C}$ for 45 s, extension at $72{ }^{\circ} \mathrm{C}$ for $1 \mathrm{~min}$ and final extension at $72{ }^{\circ} \mathrm{C}$ for $2 \mathrm{~min}$. Amplified products were detected by agarose gel electrophoresis using $2.0 \%$ agarose $(\mathrm{w} / \mathrm{v})$ in TAE buffer for $2 \mathrm{~h}$ at $70 \mathrm{~V}$. E. faecalis strain E206 (vanA) and E. faecium E2781 (vanB) kindly provided by Dr N. Woodford were used as controls in the PCR experiments.

PFGE of vancomycin-resistant enterococci. Cell preparation for PFGE was performed as described previously (Morrison et al., 1999). Each of the blocks was digested with $20 \mathrm{U}$ of SmaI restriction endonuclease (New England Biolabs) and incubated at $25^{\circ} \mathrm{C}$ for $4 \mathrm{~h}$. Electrophoresis was performed with the CHEF DR-III system (Bio-Rad) with the following run parameters: initial pulse, $5 \mathrm{~s}$; final pulse, $40 \mathrm{~s}$; voltage, $6 \mathrm{~V} \mathrm{~cm}^{-1}$; time, $20 \mathrm{~h}$; and temperature, $10^{\circ} \mathrm{C}$. The gel was stained in ethidium bromide solution $\left(0.5 \mu \mathrm{g} \mathrm{ml}^{-1}\right)$ and visualized with UV transillumination. Banding patterns were compared visually and isolates that had the same number of fragments of the same size were regarded as being the same strain.

Transfer of vancomycin resistance. Donor strains were mixed with E. faecalis recipient strain $\mathrm{JH} 2-2$ on filter membranes as described previously (Woodford et al., 1991) and transconjugants were selected on BHI agar containing $100 \mu \mathrm{g}$ rifampicin, $32 \mu \mathrm{g}$ fusidic acid, $32 \mu \mathrm{g}$ vancomycin or $15 \mu \mathrm{g}$ erythromycin. Transconjugants were screened for the co-transfer of unselected markers by replica plating. Representative colonies were analysed for the carriage of plasmid DNA as described previously (Werner et al., 1999).

\section{RESULTS}

A total of 415 enterococcal isolates were obtained from different clinical samples in five hospitals in Kuwait. The majority of them were obtained from urine $(36.6 \%)$, stool $(11.8 \%)$, wound swabs $(11.0 \%)$, blood $(10.3 \%)$, high vaginal swabs $(9 \cdot 1 \%)$, cervical swabs $(3 \cdot 1 \%)$ and miscellaneous sources, including cervical swabs, catheter tips, endotracheal secretions and ear swabs $(18.0 \%)$. The stool samples were used to screen for faecal carriage of VRE in Intensive Care Unit patients who had been on prolonged therapy with vancomycin and were suspected to suffer from
Clostridium difficile associated diarrhoea. The isolates were identified as E. faecalis ( $85 \cdot 3 \%)$, Enterococcus faecium (7.7 $\%)$, Enterococcus casseliflavus (4.0\%), Enterococcus avium $(1.2 \%)$, Enterococcus durans (1.0\%), Enterococcus gallinarium $(0.5 \%)$ and 'Enterococcus bovis' $(0.2 \%)$.

\section{Antibacterial resistance}

The distribution of antibacterial resistance according to species is presented in Table 1 . They were resistant to erythromycin $(63.3 \%)$, tetracycline $(60.5 \%)$, ciprofloxacin $(40.0 \%)$ and chloramphenicol $(28.0 \%)$. High-level resistance to gentamicin (MIC $\geqslant 500 \mu \mathrm{g} \mathrm{ml}^{-1}$ ), kanamycin (MIC $\geqslant 1000 \mu \mathrm{g} \mathrm{ml}^{-1}$ ) and streptomycin (MIC $\geqslant 2000 \mu \mathrm{g} \mathrm{ml}^{-1}$ ) was detected in $13.9,18.8 \%$ and $20.0 \%$ of the isolates respectively. In addition, $52(12.5 \%)$ of the isolates were resistant to ampicillin (MIC $\geqslant 16 \mu \mathrm{g} \mathrm{ml}^{-1}$ ). However, none of them produced $\beta$-lactamase.

The isolates were tested for their susceptibility to linezolid, a new oxazolidinone antibacterial that has been reported to have activity against Gram-positive cocci, including methicillin-resistant $S$. aureus and VRE. They were all susceptible to linezolid (MIC range: $0 \cdot 5-4 \mu \mathrm{g} \mathrm{ml}^{-1}$ ).

Vancomycin and teicoplanin resistance were detected in 11 $(2.6 \%)$ isolates consisting of four E. faecium and seven $E$. faecalis (Table 1). Six of the seven vancomycin-resistant $E$. faecalis and all four vancomycin-resistant E. faecium isolates had MIC values $>256 \mu \mathrm{g} \mathrm{ml}^{-1}$ for vancomycin (Table 2). Three E. faecalis and two E. faecium had MIC values $>256 \mu \mathrm{g}$ $\mathrm{ml}^{-1}$ for teicoplanin. All of the vancomycin-resistant isolates expressed low MIC values for linezolid (MIC $\leqslant 2 \mu \mathrm{g} \mathrm{ml}^{-1}$ ). One E. casseliflavus and two E. gallinarium isolates had MIC $8 \mu \mathrm{g} \mathrm{ml}^{-1}$ for vancomycin (Table 2). The vancomycinresistant isolates were also resistant to erythromycin, ciprofloxacin and high-level streptomycin. Six of them were resistant to ampicillin, nine isolates expressed high-level gentamicin resistance, four were resistant to tetracycline and one was resistant to chloramphenicol (Table 2).

\section{Detection of van genotypes}

The 11 vancomycin-resistant enterococci were investigated for their vancomycin-resistance genotypes by PCR. All of them gave positive results for the presence of the vanA genotype (Fig. 1a and Table 2). A 732 bp PCR product (Dutka-Malen et al., 1995) was obtained in all the positive isolates. No $\operatorname{van} B$ products were detected in any of the isolates (Fig. 1b).

\section{Transfer of vancomycin resistance}

Four vancomycin-resistant isolates, E. faecium E166 and E194 and E. faecalis E90 and E178, were selected on the basis of their resistance patterns and used as donors in separate filter mating experiments. Transconjugants were obtained only with E. faecalis E90 as the donor. Transconjugants were obtained on vancomycin as well as on erythromycin selection plates at frequencies of $6.0 \times 10^{-6}$ c.f.u. $\mathrm{ml}^{-1}$. The trans- 
Table 1. Antibacterial resistance of enterococci by disk diffusion tests

\begin{tabular}{|c|c|c|c|c|c|}
\hline \multirow[t]{2}{*}{ Antibacterial } & \multirow{2}{*}{$\begin{array}{l}\text { MIC breakpoint } \\
\quad\left(\mu \mathrm{g} \mathrm{ml}^{-1}\right)\end{array}$} & \multicolumn{4}{|c|}{ Resistant isolates (\%) } \\
\hline & & $\begin{array}{c}\text { E. faecalis } \\
(n=354)\end{array}$ & $\begin{array}{l}\text { E. faecium } \\
(n=32)\end{array}$ & $\begin{array}{c}\text { Other } \\
\text { species* }^{*} \\
(n=29)\end{array}$ & $\begin{array}{c}\text { Total } \\
(n=415)\end{array}$ \\
\hline Ampicillin & $>16$ & $29(8 \cdot 2)$ & $15(46 \cdot 8)$ & $8(27 \cdot 6)$ & $52(12 \cdot 5)$ \\
\hline Gentamicin & $>500$ & $52(14 \cdot 7)$ & $5(15 \cdot 6)$ & $1(3 \cdot 4)$ & $58(13 \cdot 9)$ \\
\hline Kanamycin & $>1000$ & $72(20 \cdot 3)$ & $5(15 \cdot 6)$ & $1(3 \cdot 4)$ & $78(18 \cdot 8)$ \\
\hline Streptomycin & $>2000$ & $76(21 \cdot 5)$ & $6(18 \cdot 7)$ & - & $83(20 \cdot 0)$ \\
\hline Erythromycin & ND & $235(66 \cdot 4)$ & $16(50 \cdot 0)$ & $12(41 \cdot 3)$ & $263(63 \cdot 3)$ \\
\hline Tetracycline & $\mathrm{ND}$ & $229(64 \cdot 6)$ & $13(40 \cdot 6)$ & $5(17 \cdot 2)$ & $251(60 \cdot 5)$ \\
\hline Ciprofloxacin & $\mathrm{ND}$ & $140(39 \cdot 5)$ & $16(50 \cdot 0)$ & $10(34 \cdot 4)$ & $166(40 \cdot 0)$ \\
\hline Chloramphenicol & $\mathrm{ND}$ & $106(29 \cdot 9)$ & $7(21 \cdot 8)$ & $3(10 \cdot 3)$ & $116(27 \cdot 9)$ \\
\hline Vancomycin & $>32$ & $7(1 \cdot 9)$ & $4(12 \cdot 5)$ & - & $11(2 \cdot 6)$ \\
\hline Teicoplanin & $>32$ & $7(1 \cdot 9)$ & $4(12 \cdot 5)$ & - & $11(2 \cdot 6)$ \\
\hline
\end{tabular}

ND, MIC not determined.

* Other species consisted of E. casseliflavus ( $n=17)$, E. avium (5), E. durans (4), E. gallinarium (2) and 'E. bovis' (1).

\section{Table 2. Antibacterial-resistance profiles and van genotypes of VRE isolates}

Abbreviations: Amp; ampicillin, Cm, chloramphenicol, Cip, ciprofloxacin, Em, erythromycin, Gm, gentamicin (high-level), Sm, streptomycin (highlevel), Tc, tetracycline. +, Positive, -, negative. Isolates E166, E194, E246 and E249 were from one hospital while the other isolates were from another hospital. Strains XU11 and XU15 were control strains for vanA and vanB genes, respectively, kindly provided by Dr N. Woodford.

\begin{tabular}{|c|c|c|c|c|c|c|c|}
\hline \multirow[t]{2}{*}{ Isolate } & \multirow[t]{2}{*}{ Source } & \multirow[t]{2}{*}{ Species } & \multicolumn{2}{|c|}{$\operatorname{MIC}\left(\boldsymbol{\mu g ~ m l} \mathbf{l}^{-1}\right)$} & \multicolumn{2}{|c|}{ Genotype } & \multirow[t]{2}{*}{ Resistance pattern } \\
\hline & & & Van & Tec & $\operatorname{van} A$ & $\operatorname{van} B$ & \\
\hline E166 & Urine & E. faecium & $>256$ & $>256$ & + & - & Amp, Em, Cip, Gm, Sm \\
\hline E194 & Urine & E. faecium & $>256$ & $>256$ & + & - & Em, Cip, Gm, Sm, Cm \\
\hline E232 & Wound & E. faecalis & $>256$ & 256 & + & - & Em, m, Cip, Gm, Sm \\
\hline E246 & Urine & E. faecalis & $>256$ & 32 & + & - & Amp, Em, Cip, Gm, Sm, Tc \\
\hline E249 & Wound & E. faecalis & $>256$ & $>256$ & + & - & Em, Cip, Gm, Sm, Tc \\
\hline V254 & Wound & E. faecium & $>256$ & 24 & + & - & Amp, Em, Cip, Gm, Sm \\
\hline V261 & Blood & E. faecalis & $>256$ & $>256$ & + & - & Amp, Em, Cip, Gm, Sm \\
\hline XU11 & & E. faecalis E206 & $>256$ & $>256$ & + & - & $\mathrm{ND}$ \\
\hline XU15 & & E. faecium $\mathrm{E} 2781$ & 64 & 1.5 & - & + & ND \\
\hline
\end{tabular}

conjugants from both selections were all resistant to vancomycin, teicoplanin, erythromycin, kanamycin and streptomycin, but no detectable plasmid was demonstrated in any of the transconjugants or the parental strain. The vanA product of the same size as that in the parent strain was obtained when genomic DNA from representative transconjugants was tested by PCR (Fig. 1a).

\section{PFGE of vancomycin-resistant isolates}

The vancomycin-resistant isolates were typed by PFGE after $S m a I$ digestion of their genomic DNA. Two of the seven $E$. faecalis isolates had identical PFGE patterns as shown in Fig. 2. These isolates were obtained from different patients who had been admitted to different wards in the same hospital. 
(a)

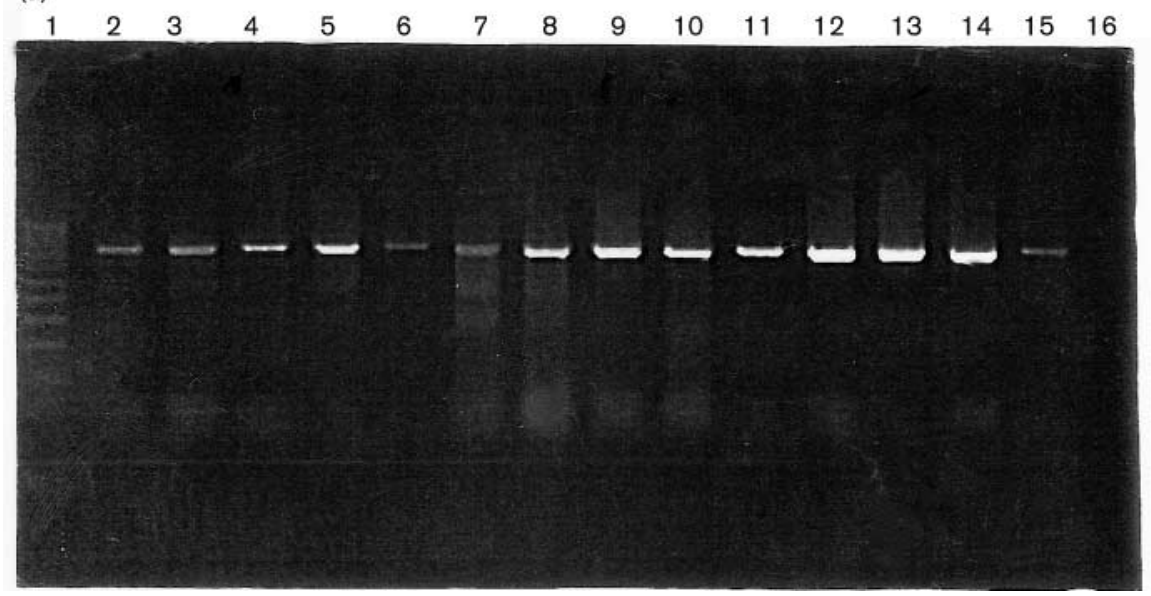

(b)

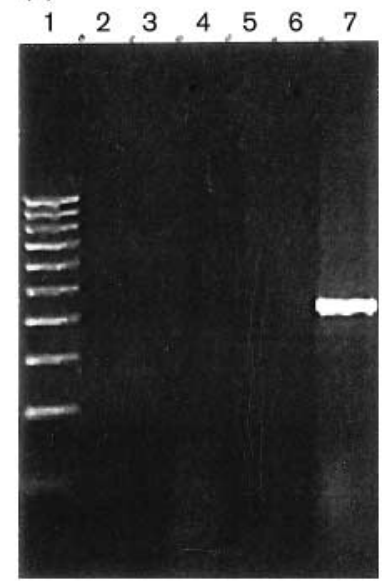

Fig. 1. PCR amplification of (a) vanA and (b) vanB genes. (a) Lanes: 1, molecular size markers; 2-8; vancomycin-resistant E. faecalis isolates V90, E178, E232, E246, E249, V261 and V296; 9- 12; vancomycin-resistant E. faecium isolates, E165, E166, E194 and V254; 13-14, representatives of E90 vancomycin-resistant transconjugants; 15, E. faecalis E206, vanA positive control strain; 16; E. faecalis ATCC 29212, vancomycinsusceptible strain. All of the vancomycin-resistant isolates generated a 732 bp PCR product. (b) Lanes: 1, Molecular size markers; 2-6; representatives of vancomycin-resistant strains; 7, E. faecium E2781, vanB positive control strain.

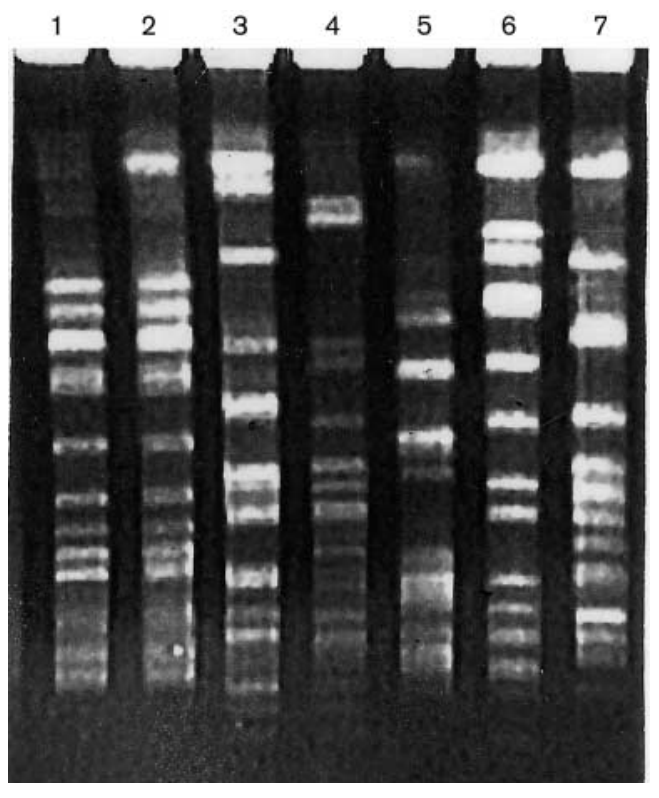

Fig. 2. PFGE patterns of seven vancomycin-resistant E. faecalis isolates digested with Smal restriction endonuclease. Lanes: 1, E246; 2, E249; 3, V90; 4, E178; 5, E232; 6, V261; 7, V296.

One isolate was from a urine sample and the other was from a diabetic foot wound swab. The remaining five E. faecalis isolates had different PFGE patterns (Fig. 2). Similarly, the four E. faecium isolates had different PFGE patterns (not shown).

\section{DISCUSSION}

This study investigated the species prevalence and antibacterial-resistance patterns of enterococci isolated from clinical samples in Kuwait hospitals. The majority of the isolates were either E. faecalis $(85.3 \%)$ or E. faecium $(7 \cdot 7 \%)$, while E. casseliflavus, E. avium, E. gallinarium, E. durans and ' $E$. bovis' accounted for only $7 \cdot 0 \%$ of the isolates, which was comparable to the distribution of enterococcal species in other studies (Jones et al., 1997; Perlada et al., 1997; Zouain \& Araj, 2001). The prevalence of E. faecium in this study (7.7 \%) was lower than a $29 \%$ prevalence of E. faecium reported in Cincinnati, OH, USA (Perlada et al., 1997). E. faecium has also been reported as the most common species in other studies (Woodford et al., 1993; Linden et al., 1996). No VRE were isolated from any of the stool samples submitted for the detection of $C$. difficile toxin in this study in contrast to the detection of VRE in $19 \cdot 8 \%$ of stool samples screened for $C$. difficile toxin by Leber et al. (2001).

Of the 415 isolates, 63, 60 and $40 \%$ were resistant to erythromycin, tetracycline and chloramphenicol, respectively which were similar to levels reported for these antibacterials among enterococci isolated in the UK (Woodford et al., 1993) and Germany (Reinert et al., 1999). It was reassuring that 98 and $91.8 \%$ of the E. faecalis in this study were vancomycin- and ampicillin-susceptible, respectively, contrary to the situation in most hospitals in the USA (Jones et al., 1997; Quale et al., 1996; Perlada et al., 1997) and Europe (Nelson et al., 2000; Schouten et al., 2000) where high prevalence of vancomycin and ampicillin resistance are common. The low prevalence of vancomycin resistance 
among the isolates in this study indicated that vancomycin retains its therapeutic efficacy against the majority of enterococci isolated from patients in Kuwait hospitals.

Although 85.3 and $84.5 \%$ of the isolates were susceptible to gentamicin and streptomycin, suggesting that gentamicin should maintain a synergistic effect when combined with cell-wall-active agents such as vancomycin and ampicillin in the treatment of enterococcal infections, the detection of high-level gentamicin resistance in $14.7 \%$ of E. faecalis and $15.6 \%$ of E. faecium isolates is cause for concern, as it may signify the beginning of a major resistance problem.

Vancomycin-resistance phenotypes in enterococci have been classified as vanA, vanB, vanC, vanD and $v a n E$ based on levels of resistance, cross-resistance to teicoplanin and inducible or constitutive nature of the resistance (Arthur \& Courvalin, 1993). Two of these, vanA (vancomycin MIC $\leqslant 64 \mu \mathrm{g} \mathrm{ml}^{-1}$; teicoplanin MIC $\geqslant 16 \mu \mathrm{g} \mathrm{ml}^{-1}$ ) and vanB (vancomycin MIC $4-1024 \mu \mathrm{g} \mathrm{ml}^{-1}$, teicoplanin MIC $\left.\leqslant 0.5 \mu \mathrm{g} \mathrm{ml}^{-1}\right)$ determinants have been described primarily in $E$. faecalis and $E$. faecium. The vanA determinant is carried on transposon Tn1546 or close relatives that are transferable in conjugation experiments (Arthur \& Courvalin, 1993). All of the 11 vancomycin-resistant isolates in this study expressed vancomycin-resistance patterns compatible with the vanA phenotype and all gave positive results in PCR experiments for the vanA genotype. One of the four isolates tested transferred vancomycin resistance in conjugation experiments but the transconjugants contained no detectable plasmids, suggesting that the vancomycin-resistance determinant of this isolate may be carried on a conjugative transposon (Arthur \& Courvalin, 1993). The transfer of vancomycin resistance without the transfer of detectable plasmids has been reported previously (Shlaes et al., 1989; Leclercq et al., 1989).

PFGE revealed that only two E. faecalis isolates were related (Fig. 2). The rest were unrelated, suggesting that the strains acquired vancomycin resistance independently, possibly, by horizontal transfer of the vancomycin-resistance determinants. The absence of a dominant clone among the vancomycin-resistant isolates is consistent with the fact that there has been no evidence of an outbreak of VRE in any of the hospitals from where the isolates were obtained. Furthermore, VRE have been isolated irregularly in these hospitals.

Although the prevalence of glycopeptide resistance was low among the isolates studied, their presence together with high-level aminoglycoside resistance calls for regular surveillance of antibacterial susceptibilities to detect emerging resistance and prevent the establishment and spread of multiply antibacterial-resistant strains.

\section{ACKNOWLEDGEMENTS}

This project was funded by grant MI 111 from Kuwait University Research Administration. We thank Pharmacia Corporation for the gift of linezolid powder, Dr N. Woodford for the gift of vanA and vanB containing strains and Mrs Preethi John for technical assistance.

\section{REFERENCES}

Arthur, M. \& Courvalin, P. (1993). Genetics and mechanisms of glycopeptide resistance in enterococci. Antimicrob Agents Chemother 37, 1563-1571.

Cetinkaya, Y., Falk, P. \& Mayhall, C. G. (2000). Vancomycin-resistant enterococci. Clin Microbiol Rev 13, 686-707.

Donskey, C. J., Schreiber, J. R., Jacobs, M. R., Sheker, R., Salata, R. A., Gordo, S., Whalen, C. C., Smith, F. \& Rice, L. B. (1999). A polyclonal outbreak of predominantly vanB vancomycin-resistant enterococci in North East Ohio. Northeast Ohio vancomycin-resistant enterococcus surveillance program. Clin Infect Dis 29, 573-579.

Dutka-Malen, S., Evers, S. \& Courvalin, P. (1995). Detection of glycopeptide resistance and identification to the species level of clinically relevant enterococci by PCR. J Clin Microbiol 33, 24-27.

Huycke, M. M., Sahm, D. F. \& Gilmore, M. S. (1998). Multiple-drug resistant enterococci: The nature of the problem and an agenda for the future. Emerg Infect Dis 4, 239-249.

leven, M., Vercauteren, E., Deschemaeker, P., Van Laer, F. \& Goossens, H. (1999). Comparison of direct plating and broth enrichment culture for the detection of intestinal colonisation by glycopeptideresistant enterococci among hospitalized patients. J Clin Microbiol 37, 1436-1440.

Jones, R. N., Marshall, S. A., Pfaller, M. A., Wilke, W. W., Hollis, R. J., Erwin, M. E., Edmond, M. B. \& Wenzel, R. P. (1997). Nosocomial enterococcal bloodstream infections in the SCOPE program: antimicrobial resistance, species occurrence, molecular testing results and laboratory testing accuracy. SCOPE Hospital Study Group. Diagn Microbiol Infect Dis 29, 95-102.

Jordens, J. Z., Bates, J. \& Griffiths, D. J. (1994). Fecal carriage and nosocomial spread of vancomycin-resistant Enterococcus faecium. J Antimicrob Chemother 34, 515-528.

Leber, A. I., Hindler, J. F., Kato, E. O., Bruckner, D. A. \& Peques, D. A. (2001). Laboratory based surveillance for vancomycin-resistant enterococci: utility of screening stool specimens submitted for Clostridium toxin assay. Infect Control Hosp Epidemiol 22, 160-164.

Leclercq, R., Derlot, E., Weber, M., Duval, J. \& Courvalin, P. (1989). Transferable vancomycin and teicoplanin resistance in Enterococcus faecium. Antimicrob Agents Chemother 33, 10-15.

Linden, P. K., Pasculle, A. W., Manez, R., Kramer, D. J., Fung, J. J., Pinna, A. D. \& Kusne, S. (1996). Differences in outcomes for patients with bacteremia due to vancomycin-resistant Enterococcus faecium or vancomycin susceptible E. faecium. Clin Infect Dis 22, 663-670.

Miele, A., Bandera, M. \& Goldstein, B. P. (1995). Use of primers selective for vancomycin resistance genes to determine van genotypes in enterococci and to study gene organisation in vanA isolates. Antimicrob Agents Chemother 39, 1772-1778.

Morrison, D., Woodford, N., Barrett, S. P., Sisson, P. \& Cookson, B. D. (1999). DNA banding pattern polymorphism in vancomycin-resistant Enterococcus faecium and criteria for defining strains. J Clin Microbiol 37, 1084-1091.

National Committee for Clinical Laboratory Standards (2000). Performance standard for disk susceptibility tests. Approved Standard 7th edn. Wayne, PA: National Committee for Clinical Laboratory Standards.

Nelson, R. R. S., McGregor, K. F., Brown, A. R., Amyes, S. G. B. \& Young, H.-K. (2000). Isolation and characterization of glycopeptide-resistant enterococci from hospitalized patients over a 30 -month period. J Clin Microbiol 38, 2112-2116.

Perlada, D. E., George, S. A. \& Cushion, M. T. (1997). Molecular epidemiology and antibiotic susceptibility of enterococci in Cincinnati, Ohio: a prospective citywide survey. J Clin Microbiol 35, 2342-2347. 
Quale, J., Laudman, D., Atwood, E. \& 9 other authors (1996). Experience with a hospital-wide outbreak of vancomycin-resistant enterococci. Am J Infect Control 24, 372-379.

Reinert, R. R., Conrad, G., Schaeger, J. J., Werner, G., Witte, W., Lutticken, R. \& Klare, I. (1999). Survey of antibiotic resistance among enterococci in North Rhine-Wesphalia, Germany. J Clin Microbiol 37, $1638-1641$.

Schouten, M. A., Hoogkamp-Korstanje, J. A., Meis, J. F. \& Voss, A. (2000). Prevalence of vancomycin-resistant enterococci in Europe. Eur J Clin Microbiol Infect Dis 19, 816-822.

Shlaes, D. M., Al-Obeid, S., Shlaes, J. H., Boisivon, A. \& Williamson, R. (1989). Inducible, transferable resistance to vancomycin in Enterococcus faecium, D399. J Antimicrob Chemother 23, 503-508.

Toutouza, M., Skandami, V., Poujiouka-Bei, M., Fakiri, H., Karabassi, V., Komniou, (2001). Resistance phenotypes in enterococci isolated from clinical specimens during a 3-year period. Clin Microbiol Infect 7 (Suppl. 1), 81.
Udo, E. E. \& Dashti, A. A. (2000). Detection of genes encoding aminoglycoside modifying enzymes in Staphylococci by polymerase chain reaction and dot blot hybridization. Int J Antimicrob Agents 13, 273-279.

Werner, G., Klare, I. \& Witte, W. (1999). Large conjugative vanA plasmids in vancomycin-resistant Enterococcus faecium. J Clin Microbiol 37, $2383-2384$.

Woodford, N., Johnson, A. P. \& George, R. C. (1991). Detection of glycopeptide resistance in clinical isolates of Gram-positive bacteria. J Antimicrob Chemother 28, 483-486.

Woodford, N., Morrison, D., Johnson, A. P. \& George, R. C. (1993). Antimicrobial resistance amongst enterococci isolated in the United Kingdom: a reference laboratory perspective. J Antimicrob Chemother 32, 344-346.

Zouain, M. G. \&, Araj, G. F. (2001). Antimicrobial resistance of Enterococci in Lebanon. Int J Antimicrob Agents 17, 209-213. 